\title{
Global Simulation of the Jovian Magnetosphere: Transitional Structure From the lo Plasma Disk to the Plasma Sheet
}

\section{$\operatorname{AUTHOR}(\mathrm{S}):$}

Tanaka, T.; Ebihara, Y.; Watanabe, M.; Fujita, S.; Kataoka, R.

\section{CITATION:}

Tanaka, T....[et al]. Global Simulation of the Jovian Magnetosphere: Transitional Structure From the Io Plasma Disk to the Plasma Sheet. Journal of Geophysical Research: Space Physics 2021, 126(6): e2021JA029232.

ISSUE DATE:

2021-06

URL:

http://hdl.handle.net/2433/267747

\section{RIGHT:}

(C) 2021. American Geophysical Union. All Rights Reserved.; The full-text file will be made open to the public on 10 December 2021 in accordance with publisher's 'Terms and Conditions for Self-Archiving'. 


\section{JGR Space Physics}

\section{RESEARCH ARTICLE \\ 10.1029/2021JA029232 \\ Key Points: \\ - Io plasma near $10 \mathrm{R}_{\mathrm{J}}$ diffuses outward due to the instability effect and beyond $20 R_{\mathrm{J}}$ moves outward by centrifugal force \\ - Io plasma and the ionospheric plasma mix in the region between $15 \mathrm{R}_{\mathrm{J}}$ and $20 \mathrm{R}_{\mathrm{J}}$ and then migrate outward together \\ - Reaction of plasmoid ejection affects the instability in the Io disk causing modulation for radial plasma transport}

\section{Supporting Information:}

Supporting Information may be found in the online version of this article.

Correspondence to:

T. Tanaka,

takashi.tanaka.084@m.kyushu-u.ac.jp

Citation:

Tanaka, T., Ebihara, Y., Watanabe, M., Fujita, S., \& Kataoka, R. (2021) Global simulation of the Jovian magnetosphere: Transitional structure from the Io plasma disk to the plasma sheet. Journal of Geophysical Research: Space Physics, 126, e2021JA029232. https://doi.org/10.1029/2021JA029232

Received 13 MAR 2021 Accepted 10 MAY 2021

(C) 2021. American Geophysical Union. All Rights Reserved.

\section{Global Simulation of the Jovian Magnetosphere: Transitional Structure From the Io Plasma Disk to the Plasma Sheet}

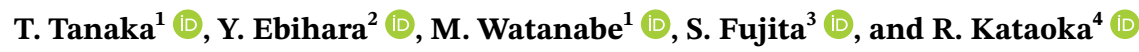 \\ ${ }^{1}$ International Center for Space Weather Science and Education, Kyushu University, Fukuoka, Japan, ${ }^{2}$ Research \\ Institute for Sustainable Humanosphere, Kyoto University, Uji, Kyoto, Japan, ${ }^{3}$ The institute of statistical mathematics, \\ Tachikawa, Tokyo, Japan, ${ }^{4}$ Space and Upper Atmospheric Group, National Institute of Polar Research, Tachikawa, \\ Tokyo, Japan
}

Abstract Jupiter has a strong magnetic field, and a huge magnetosphere is formed through the solar wind-Jupiter interaction. The generated magnetosphere-ionosphere system is reproduced based on the 9-component Magnetohydrodynamics (MHD) and the current conservation in the ionosphere. Assuming Io plasma emission rate $1.4 \mathrm{t} / \mathrm{sec}$, this paper reproduces self-consistently global magnetic configuration, generations of the field-aligned current (FAC) and aurora, formation of the Io plasma disk at 8-20 $\mathrm{R}_{\mathrm{J}}$, plasma corotation, instability in the plasma disk, transition from the Io plasma disk to the plasma sheet at 20-150 $\mathrm{R}_{\mathrm{J}}$, and the plasmoid ejection. The rotating Io plasma in the disk forms instabilities that promotes radial diffusion. $\mathrm{H}^{+}$is supplied from the ionosphere along high-latitude magnetic field lines and mixed with heavy ions around $15-20 \mathrm{R}_{\mathrm{J}}$. Beyond $20 \mathrm{R}_{\mathrm{J}}$, mixed plasma diffuses further outward by the centrifugal force that can exceed magnetic tension. In the ionosphere, the main oval occurs at $13.7^{\circ}-15.5^{\circ}$ colatitude. The Io disk is inner side of magnetic field lines traced from the low-latitude edge of the main oval. Along magnetic field lines, the main oval is mapped from the outer edge of the Io disk to the entire plasma sheet accompanying rotation delay. Due to the corotation limit, convection is accompanied by plasmoid ejection. Back reaction of plasmoid ejection affects even transport process in the Io disk. The downward FAC occurs in the polar cap showing variability. The region of externally driven Dungey convection seems quite narrow.

\section{Introduction}

The structure and dynamics of the Jupiter's magnetosphere are very different from those of the earth. The planet's magnetic field is 14 times stronger than that of the earth. Such strong magnetic field and the dilute solar wind (Hanlon et al., 2004) result in the formation of a huge magnetosphere. In the Jovian magnetosphere, the average position of the bow shock is around $80 \mathrm{R}_{\mathrm{J}}$ in front of the planet (Achilleos et al., 2004; Sarkango et al., 2019). The plasma sheet forms in the similar way as the Earth, but the plasma sheet mainly consists of plasma from the moon Io. The radius of Jupiter is 11 times larger than that of the Earth. The planetary rotation period is as short as $10 \mathrm{~h}$. High-speed planetary rotation is also transmitted to Io plasma. The magnetic axis tilts about $10^{\circ}$ from the rotation axis (Smyth et al., 2011). The shape of magnetic field deviates from the dipole, with an anomaly that provides a weak magnetic field region at high latitudes in the Northern Hemisphere (Hess et al., 2011).

Io's orbit is at $5.95 \mathrm{R}_{\mathrm{J}}$, where neutral gas is released. Diffusing gas is ionized, then about half is picked up by rotating magnetic field. The amount of plasma supplied from Io is 0.26-1.4 t/sec (Delamere \& Bagenal, 2003). The plasma components from Io are $\mathrm{O}^{+}, \mathrm{O}^{++}, \mathrm{S}^{+}, \mathrm{S}^{++}, \mathrm{S}^{+++}$, and $\mathrm{Na}^{+}$(Bodish et al., 2017; Kim et al., 2020). This Io source plasma plays a major role in constructing the inner magnetosphere, in forming the magnetosphere-ionosphere (M-I) coupling system. Io plasma forms the plasma disk in the inner magnetosphere $\left(<15 \mathrm{R}_{\mathrm{J}}\right)$, and the plasma sheet in the middle tail $\left(20 \mathrm{R}_{\mathrm{J}} \sim 60 \mathrm{R}_{\mathrm{J}}\right)$. Io source plasma is predominant over these regions (Cohen et al., 2001; Radioti et al., 2005). In the distant tail ( $>600 \mathrm{R}_{\mathrm{J}}$ ), plasma originating from the solar wind is predominant (McComas et al., 2017).

In the inner magnetosphere, Io plasma accumulates and becomes unstable when the density exceeds a certain level (Southwood \& Kivelson, 1987). Through the interchange instability possibly caused by the 
centrifugal force, diffusion mechanism works to spread Io plasma outside. Radial transport by flux tube interchange process carries plasma outward, away from the planet to displace mass but not magnetic flux (Ma et al., 2016; Winglee et al., 2013). The magnetic flux tube containing cold and heavy plasma moves outward, while the magnetic flux tube containing warm and light plasma moves inward. The time required for Io plasma to diffuse outward is 10-20 days.

Unlike the Earth's magnetosphere, whose convection is driven by its coupling to the solar wind, the Jovian magnetospheric convection is driven internally by rapid planetary rotation and large internal plasma source. Diffusing outward caused by instability, Io plasma is all the time exposed to planet's rapid rotation. For Io plasma, the breakdown of corotation occurs around $20 \mathrm{R}_{\mathrm{J}}$, whereas plasma continues to diffuse outward (Khurana et al., 2004). The field-aligned current (FAC) occurs as magnetic field lines are bent in the azimuthal direction due to delay in corotation (Cowley, Bunce \& Nichols, 2003). While corotation is enforced by acquiring angular momentum through the FAC, the FAC causes aurora on the ionosphere (Hill, 2001). In such a system, the corotation enforcement current, the FAC, and the ionospheric current are interconnected to form the current system throughout the M-I coupling system (Chané et al., 2013; Cowley et al., 2005; Moriguchi et al., 2008). On Jupiter, the rotational transmission process is a major component of magnetospheric dynamics, while the Dungey convection that predominates on the earth is not the major driver (Cowley, Bunce, Stallard \& Miller, 2003).

The cause of aurora (main oval) is the upward FAC related to the M-I coupling current system. From such mechanism in the rotating magnetosphere, the main oval will occur quasi-steadily. In this case, the dynamo is in the ionosphere (Tanaka et al., 2016). It has been shown theoretically that the maintaining of Io disk corotation and the occurrence of the main oval are linked with each other (Cowley et al., 2005), and such joint structure between the corotation and aurora has been reproduced in simulations (Chané et al., 2017; Sarkango et al., 2019). For the dipole case, (colatitudes) $13^{\circ}$ and $11^{\circ}$ are mapped to radial distances of 20 $R_{J}$ and $30 R_{J}$. In the observation, the main oval is located at slightly higher latitude than Ganymede $\left(15 R_{J}\right)$, so that the main oval is expected to map to radial distance around $20 \mathrm{R}_{\mathrm{J}}$ (Vogt et al., 2017). In this way, the main oval is mapped to a place slightly distant from Ganymede, where the corotation begins to lag. Thus, $13^{\circ}$ colatitude of the oval on the ionosphere is consistent with theoretical estimation. In reality, the magnetic field is stretched by the generation of the field perpendicular current, so the size of the oval will be larger than $13^{\circ}$ (Vogt et al., 2017).

As aurora, polar cap aurora is observed in addition to main oval aurora. Polar cap aurora is highly variable and is classified into several types (Grodent, Clarke, Kim et al., 2003; Vogt et al., 2011). The active region is very dynamic and is characterized by the presence of flares, bright spots, and arc-like appearances. Some of these phenomena are expected to relate with the cusp (Bunce et al., 2004). While upward electron beam is observed above the swirl in the center of the polar cap, optical observations show that particles penetrate to low altitudes in the atmosphere (Paranicas et al., 2018). Although details are not known for the generation mechanism of the polar cap aurora, it should relate with the twisted structure of lobe magnetic fields or the formation mechanism of the cusp.

As shown hitherto, outward diffusion of Io plasma, the enforcement of corotation, and the transmission of angular momentum by the FAC self-consistently couple in the vicinity of Jupiter. However, corotation no longer holds in the farther plasma sheet, since magnetic fields cannot stretch infinitely. Ejection of plasma through the formation of plasmoids is unavoidable in this region (Fukazawa et al., 2006). Where, rotating plasma component is detached by the reconnection to discharge the magnetized plasma downstream. This internally driven convection + plasmoid detachment structure is called Vasyliunas cycle (Cowley, Bunce, Stallard \& Miller, 2003; Delamere et al., 2015; Kivelson \& Southwood, 2005; Vasyliunas, 1983). Plasmoids on the order of $\sim 25 \mathrm{R}_{\mathrm{J}}$ in scale occur every $4 \mathrm{~h}-3$ days, with a predominance in the post-midnight sector at distances of $70-120 \mathrm{R}_{\mathrm{J}}$. How far the rotational convection component extends will be related to the magnetospheric closure of the FAC and the place of return current (downward FAC). These structures result in a model in which the downward FAC returns from the outer edge of the disk (Cowley, Bunce \& Nichols, 2003) or a model in which the FAC returns to the polar cap via the distant magnetosphere (Cowley, et al., 2005; Moriguchi et al., 2008), etc. 
The question of whether an externally driven convective component, the Dungey cycle, is effective or not is another problem on Jupiter. Pu and Kivelson (1983) concluded that about 1\% of the solar wind kinetic energy is transferred to the magnetosphere. By analogy to the Earth, inflow of solar wind energy is related to external convection drive by reconnection between the solar wind magnetic field and the magnetospheric magnetic field. If there occurs Dungey cycle, it is expected in the polar cap region (Cowley, Bunce, Stallard \& Miller, 2003). It has also been estimated by Dunn et al. (2020) that fluctuations of the main oval associated with solar wind changes indicate the effect of externally driven convection. However, fluctuations are brought about not only in externally driven convection but also in internally driven convection due to region limitation effect by the solar wind (shock) (Chané et al., 2017).

In this paper, the 9-component global magnetohydrodynamic (MHD) simulation is adopted to reproduce the Jovian M-I coupling process, with inclusion of planetary rotation, Io plasma generation, ionospheric current closure. Io plasma and solar wind plasma distributions are calculated separately, and the inner boundary of the calculation region is set to approach Jupiter up to $6 \mathrm{R}_{\mathrm{J}}$ where Io orbit is located. In this paper, we reproduce self-consistently global magnetic configuration, formation of the Io plasma disk, instability in the plasma disk, plasma corotation, generations of the FAC and aurora, plasma transport from the Io plasma disk to the plasma sheet, and the plasmoid ejection.

\section{Simulation Method}

Until now, various global models have been developed for the Jovian magnetospheric simulation. In early days, the inner boundary of models was set beyond $10 \mathrm{R}_{\mathrm{J}}$. These models have clarified the formation process of the cusp (Miyoshi \& Kusano, 2001), appearance of the FAC in the ionosphere (Walker \& Ogino, 2003), and the plasmoid release process (Fukazawa et al., 2006). Moriguchi et al. (2008) set the internal boundary at $8 \mathrm{R}_{\mathrm{J}}$ with the Io plasma treated as the boundary condition and reproduced the ionospheric current, the FAC, equatorial current, magnetopause current, and the global current system connecting them. By introducing the extended ionospheric region in the radial distance from 4.5 to $8.5 \mathrm{Rj}$, Chané et al. (2013) reproduced the distribution of Io plasma and the FAC in the Jovian ionosphere from the calculation that includes the Io orbit at $6 \mathrm{R}_{\mathrm{J}}$. Sarkango et al. (2019) improved the BATSRUS model and set the inner boundary closer to Jupiter at $2.5 \mathrm{Re}$. The advanced feature here is that Io plasma is treated as a self-consistent structure. This simulation reproduced how the main oval respond to the solar wind shock. Taking the inner boundary at 4.5 Re, Chané et al. (2017) also gave the auroral respond to the solar wind shock. In addition, there is a model of multi-component calculation for Saturn (Winglee et al., 2013). This calculation distinguishes Enceladus plasma from solar wind plasma by adopting multi-component equations. This simulation reproduced the instability of the Enceladus plasma disk together with fingers extending from the disk.

In the present calculation, we adopt a revised version of the parallelized high-precision M-I coupling simulation REPPU (REProduce Plasma Universe) code (Tanaka et al., 2017). The major revision is to use the 9-component MHD as the basic equation (Tanaka \& Murawski, 1997) to distinguish heavy ions and $\mathrm{H}^{+}$. Heavy ions of Io origin include $\mathrm{O}^{+}, \mathrm{O}^{++}, \mathrm{S}^{+}, \mathrm{S}^{++}, \mathrm{S}^{+++}$, and $\mathrm{Na}^{+}$(Bodish et al., 2017). In the MHD calculation, only total mass density $(\mathrm{amu} / \mathrm{cc})$ is obtained for heavy ions, so that calculation can hold regardless of their composition. In this paper, heavy Io ions are indicated as $\mathrm{O}^{+}$on behalf of ions. However, conversion to number density or charge density depends on the composition. If all ions are $\mathrm{O}^{+}$or $\mathrm{S}^{++}$, number density and chare density are $1 / 16$ of mass density. The inner boundary is set at $6 R_{\mathrm{J}}$. The position of the inner boundary is still far from the ionosphere of the planet, but is a minimum necessary condition for calculating the structure of Io plasma. At present, it is difficult to set the inner boundary closer to Jupiter than $6 \mathrm{R}_{\mathrm{J}}$ due to the CFL condition. In addition, magnetic field strength, planetary rotation speed, tilt of the rotation axis, and tilt of the magnetic axis are adjusted to Jupiter.

In the calculation of solar wind and magnetospheric regions, directions of the XYZ coordinates are toward the sun, toward the opposite direction of the orbital motion, and toward north, respectively. We call this coordinate the solar coordinate. The 9-component MHD equation is solved in this area. This equation makes it possible to distinguish the Io plasma component (notate as $\mathrm{O}^{+}$) from $\mathrm{H}^{+}$component in the solar wind or in the ionosphere. The discretization of the space adopts the triangular grid system generated by dividing a dodecahedron and the total variation diminishing (TVD) scheme. The supporting information in Tanaka 
et al. (2020) shows the visualization of this grid system. The inner boundary of the calculation region is a sphere with a radius $6 R_{J}$, and the outer boundaries are $X=180 R_{J}$ on the solar wind side, and $X=-900 R_{J}$ on the tail side. In this paper, a constant solar wind is given at $\mathrm{X}=180 \mathrm{R}_{\mathrm{J}}$.

As the loading rate of Io plasma, the total amount is $1.4 \mathrm{t} / \mathrm{sec}$. Since the fine scale instability at the center of the Io disk cannot be calculated in this paper (Wu et al., 2007), the source region is given in a slightly wider range. The source of Io plasma is assumed to distribute inside a torus of $R=7-11 R_{J}$ and $Z= \pm 1 R_{J}$. Production rate inside the torus is given so as to be proportional to 1/R. Emitted Io plasma is assumed cold. The observed Io mass loading is 0.26-1.4 t/sec (Delamere \& Bagenal, 2003; Kimura, 2018). Therefore, assumed rate corresponds to expected upper limit. The magnetic field $\mathrm{B}$ is calculated as $\mathrm{B}=\mathrm{B}_{0}+\mathrm{B}_{1}$ by adopting potential magnetic field $\mathrm{B}_{0}$ separation (Tanaka, 1994). A rotating dipole magnetic field is applied to $\mathrm{B}_{0}$. Jupiter's axis of rotation is assumed to be tilted $5^{\circ}$ toward the Sun in the Northern Hemisphere. This axis is fixed throughout the calculation. Let the coordinate with the rotation axis as the pole (coordinates $5^{\circ}$ tilted toward the sun from the solar coordinate) be the rotation axis coordinate. Representing the Jovian magnetic field with dipoles is still an approximation. It does not perfectly represent the observed configuration. Still, to get a little closer to reality, we assume the magnetic axis that is tilted an additional $5^{\circ}$ from the axis of rotation, and a precession around the axis of rotation (Smyth et al., 2011). The coordinate fixed to $\mathrm{B}_{0}$ taking the magnetic axis as the pole is defined as the magnetic coordinate. This coordinate rotates around the rotation axis with precessions. At the start of the calculation, the magnetic coordinate is set $5^{\circ}$ tilted toward midnight in the northern hemisphere, viewed from the rotation axis coordinate.

At the inner boundary, the Neumann condition is adopted for the Io plasma component, and $\mathrm{H}^{+}$density is set to constant. The inner boundary conditions for velocity $\mathrm{V}$ is given with reference to the magnetic field $\mathrm{B}_{0} . \mathrm{V}_{\perp}$ is calculated from the rotation potential + mapped backed ionospheric potential, and the Neumann condition is adopted for $\mathrm{V}_{\|}$. The inner boundary conditions for magnetic field is given with reference to the spherical surface. $B_{1 \|}$ is set to 0 and $B_{1 \perp}$ is determined from the Neumann condition. The solar wind gives the outer boundary condition at $\mathrm{X}=180 \mathrm{R}_{\mathrm{J}}$. In the solar wind, $\mathrm{H}^{+}$density is $0.2 / \mathrm{cc}, \mathrm{O}^{+}$density is 0.0 / $\mathrm{cc}, \mathrm{Vx}=360 \mathrm{~km} / \mathrm{sec}$, and $\mathrm{Vy}=\mathrm{Vz}=0$. The interplanetary magnetic field (IMF) is $0.7 \mathrm{nT}$ with only the $\mathrm{Y}$ component. These solar wind conditions are constant over the whole calculation time. All downstream $\left(\mathrm{X}=-900 \mathrm{R}_{\mathrm{J}}\right)$ boundary conditions are the Neumann condition.

The ionosphere is set at $1 \mathrm{R}_{\mathrm{J}}$. Giving the FAC, the current continuity equation is solved to obtain the potential. In the ionosphere, the Pedersen conductivity is a function of solar zenith angle (SZA), the FAC, pressure $\mathrm{P}$, and temperature $\mathrm{T}$. The two-dimensional electric conductivity tensor $\Sigma\left(\Sigma_{\mathrm{xx}}, \Sigma_{\mathrm{xy}}, \Sigma_{\mathrm{yx}}, \Sigma_{\mathrm{yy}}\right)$ is derived from the Pedersen conductivity and constant Hall/Pedersen ratio. The FAC, P, and T are projected along $\mathrm{B}_{0}$ from $6 \mathrm{R}_{\mathrm{J}}$ to $1 \mathrm{R}_{\mathrm{J}}$. Theoretical predictions show that the Pedersen conductivity in the polar region is from 0.1 $\mho$ (background) to $7.5 \mho$ (at FAC peak $\sim 1 \mu \mathrm{A} / \mathrm{m}^{2}$ ) (Nichols \& Cowley, 2004). In the present simulation, the value of $\Sigma$ is set to be approximately the same to the Earth's case. That is, the proportional coefficient between FAC and the Pedersen conductivity is the same to the earth. Observations have shown that the swirl aurora also corresponds to the downward FAC (Paranicas et al., 2018). In consideration of this phenomena, $\Sigma$ is increased also in proportion to the downward FAC. However, the proportional coefficient is set to 0.4 times of that for the upward FAC. Similarly, the dependence of $\Sigma$ on P and T is set to be approximately the same as that of the earth. In the actual calculation result, as will be shown later, the $\Sigma_{\mathrm{xx}}$ is about $8 \mho$ at the maximum point of the FAC. For the stability of the calculation, the SZA component is slightly increased at night. The calculated potential is mapped back to the inner boundary of the MHD calculation. At the inner boundary, $\mathrm{V}_{\perp}$ is calculated after adding rotation potential. Through such feedback process, we constitute the M-I coupling system.

\section{Results and Discussion}

In the simulation of this paper, we would like to show that the obtained solution self-consistently reproduces the global magnetic configuration, distribution of Io emitted ions, distribution of ionospheric $\mathrm{H}^{+}$ions, the dynamics of the Io plasma disk and the plasma sheet, and connections of magnetic field and the FAC to the auroral ionosphere. In addition, we would like to verify the basic structure of the M-I coupling system by comparing calculated results with satellite and aurora observations. Mixing and transporting processes of 
Last-closed and first-open field lines in the solar wind-magnetosphere interaction

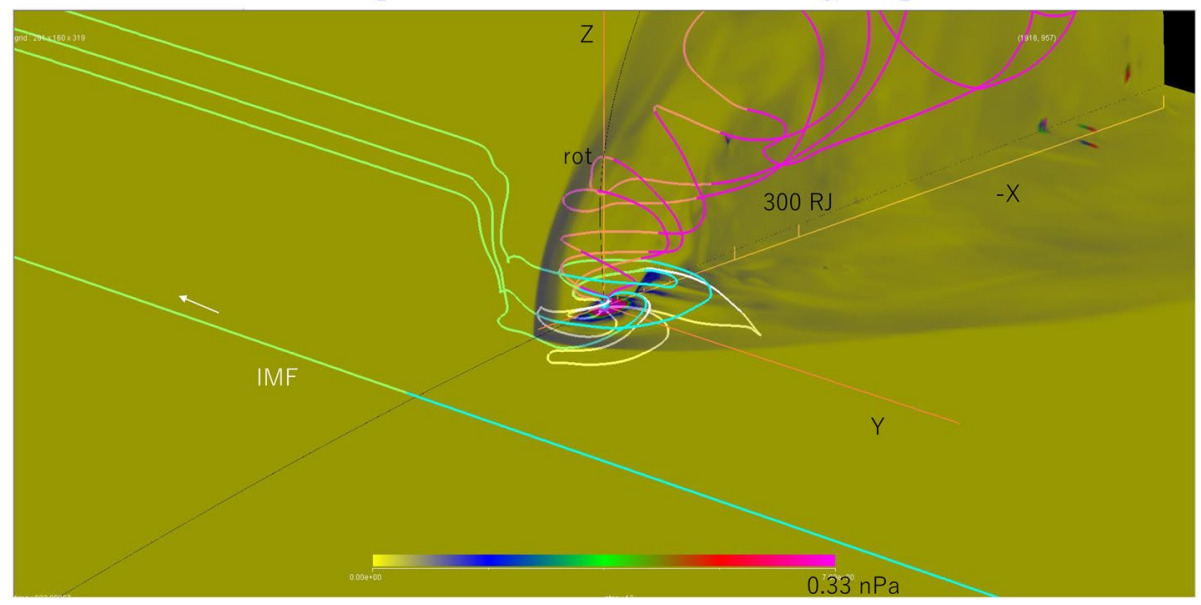

Figure 1. Last-closed (white) and first open (blue and magenta) magnetic field lines in the solar wind - Jovian magnetospheric interaction system. These field lines are on the boundary (sparatrix) between different kinds of magnetic field lines. Among first open magnetic field lines, blue lines show those connected to the interplanetary magnetic field (IMF). Transparent color shadings show pressure distribution in the equatorial and 0-12 LT meridian planes in the rotation axis coordinate.

Io plasma and ionospheric plasma depend on planetary rotation, instability, FAC intensity, and electric conductivity $\Sigma$. All of these processes are reflected in the auroral fluctuation (Nichols \& Cowley, 2003, 2004). Here all elements form together a compound system. In a compound system, comparison between theory and observation becomes meaningful by using a simulation solution which achieves self-consistency.

Morphology of aurora is a reflection of the M-I coupling dynamics both on Earth and on Jupiter. Since aurora is observable, models of the M-I coupling system can be verified from auroral observations based on this concept. Self-consistent solutions are required in such comparison between observation and theory, since the fluctuation of aurora is linked to the fluctuation of all factors related to plasma transport/distribution and magnetic field structure. Jupiter's aurora will vary with respect to solar wind conditions, the amount of Io plasma emission, and ionospheric plasma (Chané et al., 2013). For example, if the dynamic pressure fluctuates among solar wind conditions, aurora fluctuations occur through expansion or contraction of the magnetospheric region (Chané et al., 2017). Self-consistency is required to compare simulation results for such event with observations.

\subsection{Interaction Between the Solar Wind and Rotating Magnetic Field}

From solutions of the simulation, we first give an overview structure of the Jovian solar wind-magnetosphere-ionosphere coupling system. Figure 1 shows the pressure distribution (semi-transparent color shadings) and key magnetic field lines on the separatrix (magenta, blue, and white lines) for the case in which the solar wind $\mathrm{H}^{+}$density is $0.2 \mathrm{amu} / \mathrm{cc}$, the solar wind speed is $360 \mathrm{~km} / \mathrm{sec}$, and $\mathrm{O}^{+}$release from Io is $1.4 \mathrm{t} /$ sec. Here, the separatrix means the boundary surface between different kinds of magnetic field lines. Figure 1 shows pressure and magnetic field structures after about 20 rotations from the initial condition. The data shown below are all for the similar period, although not exactly at the same time. The solar wind forms the bow shock on the upstream side of the magnetosphere. From the color shading, the bow shock position is seen at $X=100 R_{J}$. In Chané et al. (2013), the shock position is at $X=75 R_{J}$, probably because the solar wind density is higher (1/cc) than in the preset calculation. In Sarkango et al. (2019), the shock position is at $X=80 \mathrm{R}_{\mathrm{J}}$, probably because the solar wind speed is a little faster $(400 \mathrm{~km} / \mathrm{sec})$ than the present calculation. The bow shock position may not be constant. Even if the solar wind is constant, time fluctuations can occur because the bow shock depends not only on the solar wind conditions but also on the plasma pressure inside the magnetosphere. This situation will be explained later. The high-pressure region continuing from the bow shock toward Jupiter is the magnetosheath, and the place where pressure becomes low again toward Jupiter is the magnetopause. 
In Figure 1, a high-pressure region develops around Jupiter. This structure seems to form with plasma originating from Io and Jupiter. In the present MHD calculation, heavy ions originating from Io can be distinguished from $\mathrm{H}^{+}$originating from the Jovian ionosphere, but it is not possible to distinguish which of $\mathrm{O}^{+}$, $\mathrm{O}^{++}, \mathrm{S}^{+}, \mathrm{S}^{++}, \mathrm{S}^{+++}$, and $\mathrm{Na}^{+}$construct heavy ions from Io. Only the sum of these ions is calculated as amu/ cc. In this paper, the sum value in amu/cc is called $\mathrm{O}^{+}$mass density for simplicity. Depending on ionic compositions, amu per charge varies between 15 and 26 (Bodish et al., 2017). However, this value is unknown from the simulation. In addition, $\mathrm{H}^{+}$originating from the ionosphere cannot be directly distinguished from $\mathrm{H}^{+}$due to the penetration of the solar wind. However, there is a possibility to distinguish them from characteristics in their distribution and their mutual interrelationships.

Figure 1 shows the last-closed and first-open magnetic field lines with some magnetic field lines in the immediate vicinity. Among them, white field lines are the last-closed magnetic field line. On the low-latitude side of these magnetic field lines, there stacked magnetic field lines having a shape close to the dipole. However, the last-closed magnetic field lines are strongly draping in the longitude direction. Closed magnetic field lines at high latitudes will naturally show the similar shape. These field lines can be understood as deformed dipole magnetic fields that bend in the direction opposite to the direction of rotation in an attempt to correct the rotational delay. On the day side, the last closed magnetic field lines extend to the vicinity of the magnetopause, and on the night side they reach as far as $150 \mathrm{R}_{\mathrm{J}}$. This structure was already shown in the simulation of Sarkango et al. (2019). Among the first-open magnetic field lines in Figure 1, there are twisted magnetic field lines (magenta) in the lobe. They extend into the lobe and reach the downstream boundary. These magenta field lines extend to the lobe away from the equatorial plane where plasma pressure is rather low. White and magenta magnetic field lines were already drawn by Miyoshi and Kusano (2001). Their shapes are almost the same as shown by Miyoshi and Kusano (2001). Not only magenta field lines but also white magnetic field lines do not reach to the equatorial plasma sheet farther than $150 \mathrm{R}_{\mathrm{J}}$. Detached field lines that occupy this area is so turbulent that no clear structure can be drawn here.

Blue field lines are open field lines between white and magenta field lines. Exactly, these blue field lines rather than magenta field lines are the first-open field lines, although their positions are very close to each other. These blue field lines pass along the magnetopause one turn around Jupiter, and then leads to the IMF in the -Y direction. Similar magnetic field lines in the Southern Hemisphere will connect to the IMF in the $+Y$ direction. Such cusp-like field line exists only in a narrow latitude width. Since magnetic field lines lead to the IMF after passing through a draping structure, configuration of magnetic field lines is not similar to the case around the earth consists of the dipole and the IMF. Compared with the calculation of the cusp structure by Miyoshi and Kusano (2001), the cusp structure becomes different due to strong draping of the magnetic field lines that construct the cusp. The difference comes from the situation in this paper that By is the main component of the IMF. Here, the magnetic field lines contact with the IMF near the magnetopause and lead to the IMF after strong draping. Miyoshi and Kusano (2001) mainly dealt with the case of the IMF directing north and south, so their structure is closer to the case of the earth than the result of this paper. If there is the Dungey cycle, it would be related to blue magnetic field lines connected to the IMF. The longitudinal width consists of blue field lines is so narrow that Dungey cycle will not occupy a meaningful area. Recently, Zhang et al. (2021) have also shown that the longitudinal width of footprint of blue field lines in Figure 1 is very narrow. It seems reasonable to draw only one Dungey cell in contact with the main oval, as in Cowley, Bunce, Stallard and Miller (2003). However, as contrary to Cowley, Bunce, Stallard and Miller (2003), the Dungey cycle will not cover a wide area in the polar cap, since most of this area is occupied by twisted magenta field lines (as will be shown in Figure 2). In addition, since magnetic field lines involved in Dungey cycle show strong draping, it is not necessary to start the Dungey cycle in the ionosphere from the day side as drawn by Cowley, Bunce, Stallard and Miller (2003).

Figure 2 shows footprints at $6 R_{J}$ of magnetic field lines drawn in Figure 1. Color shadings on a sphere at 6 $\mathrm{R}_{\mathrm{J}}$ shows the FAC, where the scale of the FAC is displayed by multiplying $6^{3}$ so as to be approximated values in the corresponding ionosphere. A figure which illustrates the latitude distribution of the FAC in the ionosphere by projecting from $6 \mathrm{R}_{\mathrm{J}}$ to $1 \mathrm{R}_{\mathrm{J}}$ will be shown later (Figure 3 ). In Figure 2, the location of the upward FAC in the ionosphere corresponds to the main oval, and the location of the downward FAC in the polar cap corresponds to the more fluctuating polar cap aurora. Similar FAC distribution is reported by Chané 


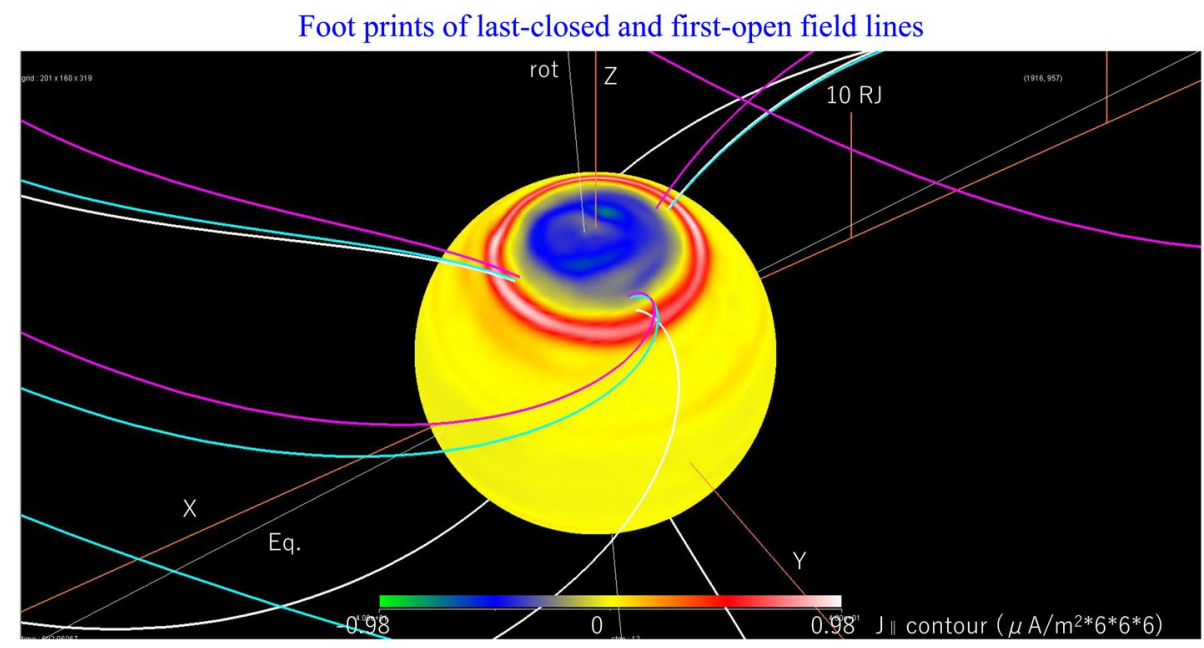

Figure 2. Foot prints of last-closed and first open field lines shown in Figure 1. Line colors are the same to Figure 1. Color shadings show distribution of the field-aligned current (FAC) on the $6 \mathrm{R}_{\mathrm{J}}$ spherical surface.

et al. (2017) and Sarkango et al. (2019). These previous papers dealt with the non-stationary solar wind, but the present paper deals only with the case of a steady solar wind.

It can be seen from Figure 2 that last-closed, first-open, and cusp magnetic field lines are all near the high-latitude edge of the main auroral oval. Therefore, formation of the main auroral oval becomes a phenomenon in the closed magnetic field line region. Connection of closed magnetic field lines to the main oval is consistent with the general understanding that the upward FAC in the auroral oval corresponds to the longitudinal deflection of closed magnetic field lines in an attempt to correct rotational delay. Also, the shape (deflection) of white field lines in Figure 1 is consistent with this understanding. It is presumed from these configurations that the simulation reproduces the corotation mechanism driven by the FAC. Here, the FAC transmits motion from the ionosphere to planetary ions captured on closed magnetic field lines connected to the oval. To be more precise, the FAC corresponds to the latitudinal difference in deflection seen in white magnetic field line. The concept that the FAC is a mechanism to transmit motion between the magnetosphere and the ionosphere is an important basic element of the magnetospheric physics (Tanaka et al., 2016).

FAC and conductivity in the ionosphere

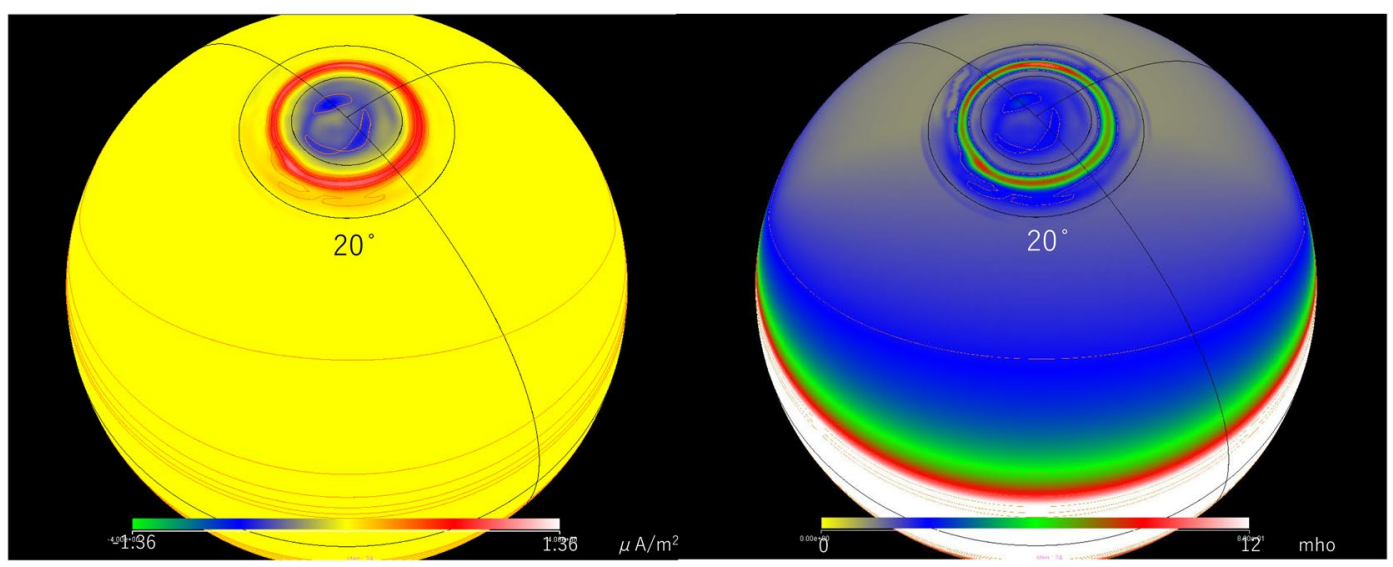

Figure 3. Field-aligned current (FAC) (left) and conductivity (right) distributions in the ionosphere at $1 \mathrm{R}_{\mathrm{J}}$ seen from the noon. Black lines on the sphere show $70^{\circ}$ and $80^{\circ}$ latitudes $\left(20^{\circ}\right.$ and $10^{\circ}$ colatitudes) of the magnetic coordinate and longitudes of 0,6 , and 18 LTs of the magnetic coordinate at the start of calculation. Conductivity shown in the right is $\Sigma_{\mathrm{XX}}$ component of integrated layer conductivity. 
The downward FAC corresponds to the twisted magnetic field lines (magenta) in the lobe. Therefore, the downward FAC is on open magnetic field lines. On the other hand, a different result is presented by Zhang et al. (2021), showing downward FAC on closed field lines. In the present paper, all magenta field lines extend far into the lobe, but in Zhang et al. (2021), many polar cap field lines connect to the opposite hemisphere passing through the evening frank. It is quite apparent that this difference is extremely serious. We think that the reason for above difference will be an issue in the future, since it is difficult to immediately clarify where the difference in these configurations comes from. Where in the magnetosphere do magnetic field lines extending from the downward and upward FAC regions in the ionosphere reach, and how does current close in the magnetosphere will be certainly the main issue in the magnetized planets. Moriguchi et al. (2008) provided an example of such current system from the simulation.

\subsection{Ionospheric Features}

Figure 3 shows the ionospheric current structure at $1 R_{\mathrm{J}}$. The left panel illustrates distribution of the FAC in the ionosphere by projecting Figure 2 down to $1 \mathrm{R}_{\mathrm{J}}$. The right panel shows distribution of $\Sigma_{\mathrm{xx}}$. Up to here, figures have been displayed in the solar coordinate, but Figure 3 is displayed in the magnetic coordinate. This figure is viewed from the noon of the solar coordinate, with black lines showing longitudes of the magnetic coordinate that were midnight, evening, and morning at the start of the calculation. The magnetic coordinate is rotating as seen in Figure 3. Latitudes drawn with black circles are $70^{\circ}$ and $80^{\circ}$ magnetic coordinates $\left(10^{\circ}\right.$ and $20^{\circ}$ colatitudes). Movie 1 of supporting information shows variation of the FAC displayed in the rotation axis coordinate for about 2 days. The black lines indicate midnight, evening, morning, and $80^{\circ}$ latitude in the rotation axis coordinate. The white line shows the longitude in the magnetic coordinate that was at midnight at the start of the calculation. In this movie, we can observe the precessional rotation of the magnetic coordinate. As far as observed from this movie, the main oval exists almost steadily. On the other hand, the downward FAC in the polar cap is highly variable.

In this paper, the electric conductivity $\Sigma$ by the aurora is set to be proportional to the upward FAC. It is also proportional to the downward FAC, but the proportional coefficient is set to 0.4 times that for the upward FAC. The proportional coefficients between $\Sigma$ and the FAC are constant in time. Since these factors are empirically determined by analogy with the Earth, results may not guarantee accuracy even if the whole is self-consistent. However, ionospheric solutions will be evaluated to a certain extent from the properness of magnetospheric solution, since the ionospheric solution is coupled with the magnetospheric solution.

In Figure 3, the center of the main oval is at $14.6^{\circ}$ colatitude. This result is almost the same as those of Chané et al. (2013) and Sarkango et al. (2019). In the present calculation, the resolution may be considerably higher than those of Chané et al. (2013) and Sarkango et al. (2019). The magnetic field lines connected to the main oval are closed magnetic field lines inside white field lines in Figure 1. At the same time, they should extend farther than magnetic field lines at the low-latitude boundary of the main oval. As will be shown later, magnetic field lines at the low-latitude boundary of the main oval extend as far as $20 \mathrm{R}_{\mathrm{J}}$, where corotation begins to delay (Chané et al., 2013). Therefore, the main oval is mapped to the plasma sheet beyond $20 \mathrm{R}_{\mathrm{J}}$ where corotation delays significantly. At the center of the main oval at $14.6^{\circ}$ colatitude, electric conductivity is $8.0 \mathrm{mho}$ and the FAC is $1.06 \mu \mathrm{A} / \mathrm{m}^{2}$. In the simulations of Chané et al. (2013) and Sarkango et al. (2019), the FAC is $0.02 \mu \mathrm{A} / \mathrm{m}^{2}$ assuming a uniform $\Sigma$ of 0.05 mho. Thus, both $\Sigma$ and FAC values in the present simulation are significantly larger than those in previous reports. Theoretical predictions suggest that there are interdependencies between Io plasma emission rate, $\Sigma$, and the FAC value (Nichols \& Cowley, 2003). Under a uniform $\Sigma$ and the Io plasma emission rate $1.5 \mathrm{t} / \mathrm{sec}$, if the electric conductivity changes between 0.1 and10 mho, the corresponding FAC becomes $0.2-20.0 \mu \mathrm{A} / \mathrm{m}^{2}$. According to this result, the FAC becomes $1 \mu \mathrm{A} / \mathrm{m}^{2}$ when the electric conductivity is about $0.5 \mathrm{mho}$. Even considering difference in Io plasma emission rate, electrical conductivity in the main oval in this paper $(8.0 \mathrm{mho})$ may be larger than theoretically estimated value. However, the electrical conductivity inside the main oval may not be so important when considering the ionospheric closure of the upward and downward FACs. What is important is the electrical conductivity near the boundary between upward and downward FACs. This value is about 2 mho in Figure 3. It will be this 2 mho that is effective when comparing the results with the case of uniform electrical conductivity. 


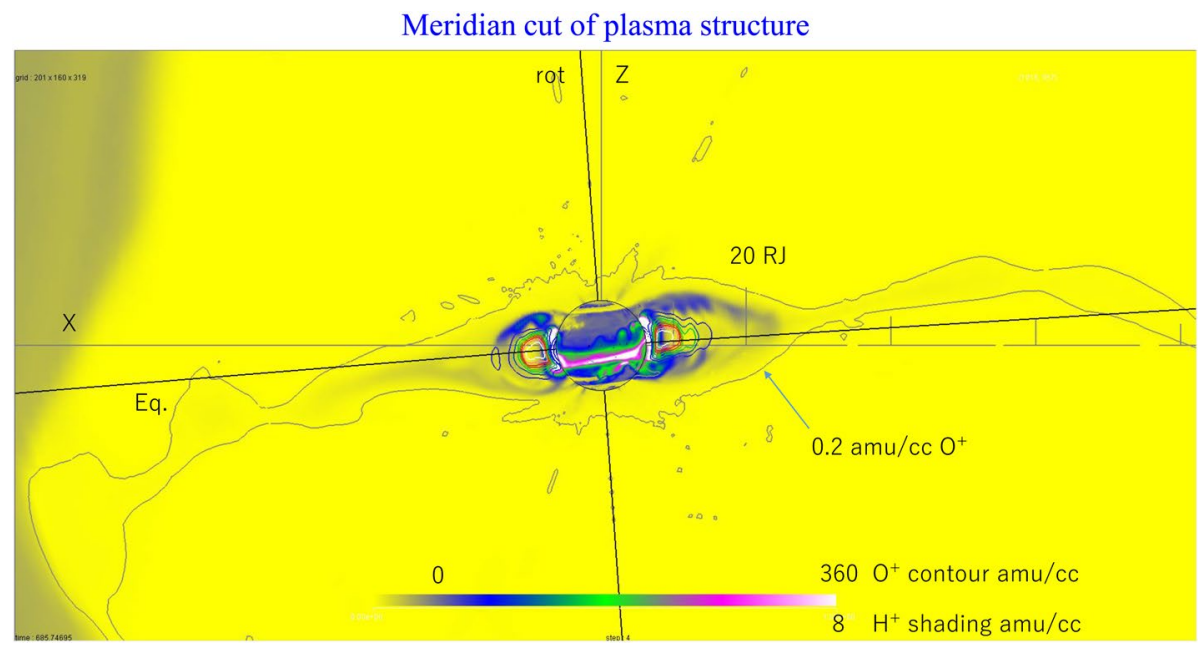

Figure 4. Meridian cut (0-12 LT) of $\mathrm{O}^{+}$(color contours) and $\mathrm{H}^{+}$(color shadings) distributions. Color shadings also show $\mathrm{H}^{+}$distribution on the spherical surface a bit above $6 \mathrm{R}_{\mathrm{J}}$.

The polar cap has the downward FAC. In the observation, overlapping with the downward FAC, the polar cap aurora occurs showing severe fluctuations. In this paper, $\Sigma$ is set to increase even in this region. The aurora with rapid fluctuations in the center of the polar cap is called the swirl (Grodent, Clarke, Kim et al., 2003). Movie 1 of the supporting information shows the fluctuating FAC in the rotation axis coordinate seen from the dayside. It can be seen from this movie that the blue part (downward FAC) fluctuates more rapidly than the red part (upward FAC). The polar cap including rapid fluctuations corresponds to the place of twisted magnetic field line (magenta lines in Figure 1). The mechanism of the polar cap aurora is not well known, but it is noteworthy to recognize that it is associated with the downward FAC. There are upward electron beams over swirl, and in the opposite side ions precipitate deep into the atmosphere (Paranicas et al., 2018). The downward FAC will be deeply involved in such particle precipitation structure.

In Movie 1, the FAC in the main oval does not show rapid fluctuations but shows only slow variations. Observations sometimes show a more intense disturbance of the main oval (Dunn et al., 2020). Such a phenomenon is often accompanied by a large solar wind disturbance, such as an arrival of a shock. In the present calculation under a steady solar wind, however, the main oval does not show severe fluctuations. Observations often show that the evening side of the main oval splits into multiple streaks (Grodent, Clarke, Waite et al., 2003). Causes of these structures are not well understood, but it is estimated from the present result that they will not be reproduced unless the solar wind is disturbed. Simulations by Sarkango et al. (2019) reproduced that the arrival of a shock causes fluctuations on the evening side of the main oval. However, the observational feature that the oval splits into multiple streaky lines has not been reproduced yet. This evening problem seems to be a next big issue. On the other hand, the polar cap FAC has large fluctuations as a whole and also includes fast fluctuations on a fine scale. This variation may be related to the swirl, but not definitive yet.

\subsection{Origin and Transport of Magnetospheric Plasma}

How solar wind plasma, ionospheric plasma, and plasma originating from Io are transported and distributed around the planet will be the main theme of Jovian magnetospheric research. Many studies have been done on this problem. Figure 4 is a zoom up view of the high-pressure region near Jupiter in Figure 1, showing XZ cross-sections of $\mathrm{O}^{+}$mass density (color contours) and $\mathrm{H}^{+}$mass density (color shadings) distributions. The color is also shown on a sphere near $6 \mathrm{R}_{\mathrm{J}}$. This part shows $\mathrm{H}^{+}$mass density distribution slightly above the internal boundary at $6 \mathrm{R}_{\mathrm{J}}$ where $\mathrm{H}^{+}$mass density is constant. High mass density regions of $\mathrm{O}^{+}$ and $\mathrm{H}^{+}$extending on the equatorial plane of the magnetic coordinate are the plasma disk and the plasma sheet. High mass density region of $\mathrm{H}^{+}$on the dayside above $70 \mathrm{R}_{\mathrm{J}}$ altitude is the magnetosheath reproduced in Figure 1. 
Instability and mixing in the equatorial plane

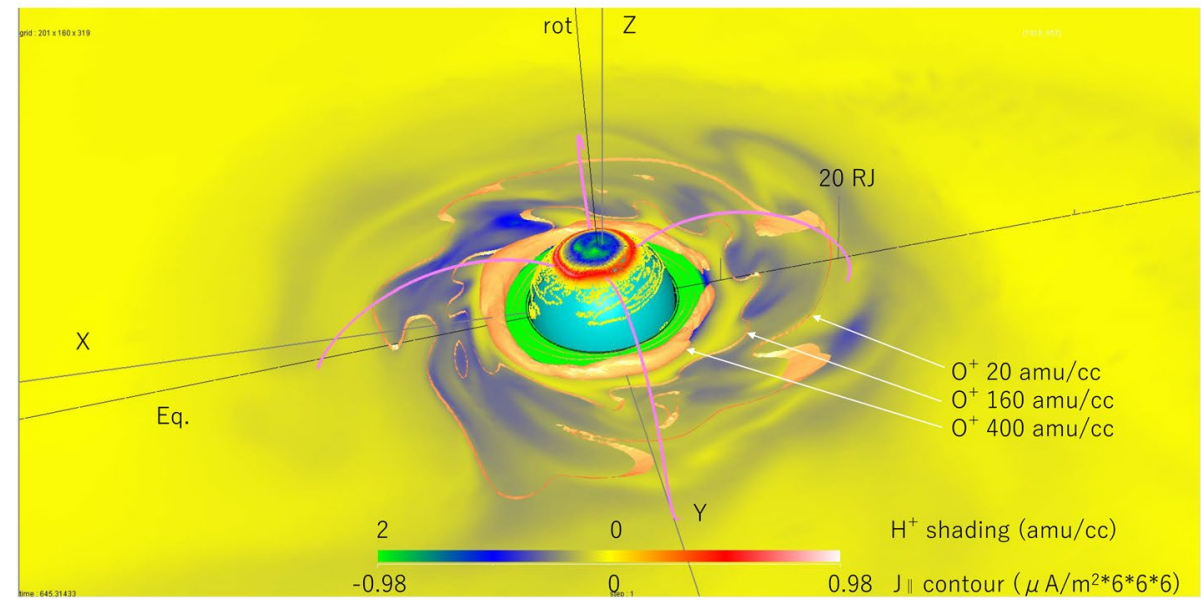

Figure 5. Distributions of $\mathrm{O}^{+}$(gold surface and contours) and $\mathrm{H}^{+}$(color shadings) mass densities in the equatorial plane of the rotational axis coordinate. Dense colored contours and pink lines show field-aligned current (FAC) distribution on the $6 \mathrm{R}_{\mathrm{J}}$ spherical surface and magnetic field lines traced from low-latitude edge of the auroral oval, respectively. Ion distributions in the equatorial plane show instability and mixing processes.

The $\mathrm{O}^{+}$distribution has a peak value above $400 \mathrm{amu} / \mathrm{cc}$ at $8 \mathrm{R}_{\mathrm{J}}$. However, distribution extending from this peak does not lead to the internal boundary. $\mathrm{O}^{+}$peak at $8 \mathrm{R}_{\mathrm{J}}$ is a reflection of the source linked to Io. Since the internal boundary is at $6 \mathrm{R}_{\mathrm{J}}$ in the present calculation, there is not enough space to reproduce actual $\mathrm{O}^{+}$distribution that accurately reflects the Io orbital at $6 \mathrm{R}_{\mathrm{J}}$. Therefore, the absolute position of the $\mathrm{O}^{+}$peak will be slightly farther away. Overall, $\mathrm{O}^{+}$is higher than $\mathrm{H}^{+}$in both mass and number densities, as is the case in observations (Cohen et al., 2001). At noon, $0.2 \mathrm{amu} / \mathrm{cc}$ contour of $\mathrm{O}^{+}$distribution extends as far as the magnetopause, but it does not mix with the solar wind $\mathrm{H}^{+}$even when it comes into contact with the magnetopause. In addition, the shape of the magnetosheath itself is also affected and deformed by $\mathrm{O}^{+}$. This effect will also lead to fluctuations in the position of the bow shock.

In the $\mathrm{H}^{+}$distribution, both mass density and number density are generally lower than those of $\mathrm{O}^{+}$. Unlike $\mathrm{O}^{+}$case, however, $\mathrm{H}^{+}$distribution continues to the ionosphere (internal boundary) at low altitudes. Looking at precisely, equatorial $\mathrm{H}^{+}$density shows a minimum around the peak of $\mathrm{O}^{+}$at $8 \mathrm{R}_{\mathrm{J}}$. In the altitude region around the $\mathrm{O}^{+}$peak, $\mathrm{H}^{+}$distributes along the magnetic field lines so as to avoid $\mathrm{O}^{+}$. Between the $\mathrm{O}^{+}$ peak and the internal boundary, $\mathrm{H}^{+}$is high pressure state in which short closed magnetic field lines at low latitudes are clogged with $\mathrm{H}^{+}$ions. Near the spherical surface, $\mathrm{H}^{+}$distribution is not uniform, but forms high-pressure areas on the side away from the equator in the rotational coordinate due to the presession effect. These regions are fairly low beta where reliability of the solution may become low because the MHD solution contains a significant amount of numerical error due to the difficulty of low beta calculation. While $\mathrm{H}^{+}$seems to distribute separately from $\mathrm{O}^{+}$along the magnetic field lines at altitudes from 10 to $20 \mathrm{R}_{\mathrm{J}}$, the spatial distribution of $\mathrm{H}^{+}$above $20 \mathrm{R}_{\mathrm{J}}$ altitude becomes similar to that of $\mathrm{O}^{+}$. Above $20 \mathrm{R}_{\mathrm{J}}, \mathrm{H}^{+}$seems to mix and coexist with $\mathrm{O}^{+}$. It can be seen from the distribution of $\mathrm{O}^{+}$that even if Jupiter $\mathrm{O}^{+}$comes into contact with the dayside magnetopause no significant mixing occurs with the solar wind within the range of MHD calculation. It seems from such situation that $\mathrm{H}^{+}$in the plasma disk and plasma sheet is mainly composed of plasma that diffuses from the ionosphere rather than that penetrated from the solar wind.

It can be estimated from these situations that both the Io disk and the plasma sheet outside the Io disk are composed mainly from $\mathrm{O}^{+}$, with an appreciable mixing of $\mathrm{H}^{+}$supplied from the ionosphere. While the inner Io disk is mainly composed of $\mathrm{O}^{+}$, the plasma sheet beyond it includes more $\mathrm{H}^{+}$. Thus it is concluded that $\mathrm{H}^{+}$in the plasma sheet beyond $20 \mathrm{R}_{\mathrm{J}}$ is supplied from the ionosphere along magnetic field lines. Mixed plasma is spreading over the entire equatorial plane of the magnetic coordinate.

Figure 5 shows $\mathrm{O}^{+}$distribution in the equatorial plane of the rotation axis coordinates (close to the $\mathrm{XY}$ plane) by gold isosurface and contour lines, similar $\mathrm{H}^{+}$distribution by color shadings, the FAC on the $6 \mathrm{R}_{\mathrm{J}}$ surface by dense color contour lines, and magnetic field lines traced from the low-latitude boundary of the 
main oval by pink lines. The drawing plane is the equatorial plane in the rotation axis coordinate that is tilted from the XY plane. However, when going to the distant tail (>80 Rj), this plane bends so that it becomes parallel to the $\mathrm{X}$ axis. This structure can be found in the supporting information of Tanaka et al. (2020). Since the magnetic axis does precession with respect to the rotation axis, the drawing plane in Figure 5 does not necessarily coincide with the center of the plasma sheet at the distant tail, but near Jupiter it can be regarded as inside the plasma sheet.

Similar to Figure 4, mass density distribution of Io emitted plasma shows a maximum value of above $400 \mathrm{amu} / \mathrm{cc}$ around $8 \mathrm{R}_{\mathrm{J}}$. With averaging wavy distribution, $160 \mathrm{amu} / \mathrm{cc}$ region spreads as far as $12 \mathrm{R}_{\mathrm{J}}$ and the $20 \mathrm{amu} / \mathrm{cc}$ region extends as far as $20 \mathrm{R}_{\mathrm{J}}$ (gold contours). In other simulation results, mass density at $20 \mathrm{R}_{\mathrm{J}}$ are $50 \mathrm{amu} / \mathrm{cc}$ (Sarkango et al., 2019) and $4 \mathrm{amu} / \mathrm{cc}$ (Chané et al., 2017). Since the fluctuation with respect to rotation is large, there are times when it spreads further and sometimes it contracts more. A gold contour with mass density of $160 \mathrm{amu} / \mathrm{cc}$ approximately corresponds to heavy ion charge density of 10/cc, but not exactly so. That is, charge density of heavy ions around $12 \mathrm{R}_{\mathrm{J}}$ is not exactly $10 / \mathrm{cc}$, because other ions than $\mathrm{O}^{+}$and $\mathrm{S}^{++}$are included. $\mathrm{H}^{+}$density originating from the ionosphere is dense on short closed magnetic field lines near the equator, but at around $10 \mathrm{R}_{\mathrm{J}}$ or above it temporarily reduces to $0.1 \mathrm{amu} / \mathrm{cc}$ or less, and then increases again to about $0.5 \mathrm{amu} / \mathrm{cc}$ at higher altitude. This tendency in Figure 5 is consistent with Figure 4. Outside $20 \mathrm{R}_{\mathrm{J}}$, the area of about $0.2 \mathrm{amu} / \mathrm{cc}\left(\mathrm{H}^{+}\right)$spreads widely. Further away (from $25 \mathrm{R}_{\mathrm{J}}$ up to $100 \mathrm{R}_{\mathrm{J}}$ ), ions originating from Io and Jupiter form a wide plasma sheet. However, the plasma sheet has a large light and shade in density.

Distributions shown by gold contour lines $\left(\mathrm{O}^{+}\right)$and colors $\left(\mathrm{H}^{+}\right)$indicate that the torus exhibits instability (exchange instability). In Figure 5, the golden torus (isosurface of $400 \mathrm{amu} / \mathrm{cc}$ ) has wrinkles and small fingers due to instability, and the $160 \mathrm{amu} / \mathrm{cc}$ contour on more outside shows clear instability. These pattern are quite similar to Kidder et al. (2009). Within $10 \mathrm{R}_{\mathrm{J}}, \mathrm{O}^{+}$and $\mathrm{H}^{+}$tend to distribute with inverse correlation. Heavy plasma of the Io torus is pushed out by centrifugal force, and the empty area is filled with light plasma outside containing $\mathrm{H}^{+}$. Conversely, at $20 \mathrm{R}_{\mathrm{J}}, \mathrm{O}^{+}$and $\mathrm{H}^{+}$distributions tend to be positively correlated. It is understood from these structure that $\mathrm{O}^{+}$diffused from inside and $\mathrm{H}^{+}$moved along magnetic fields are mixed at 10-20 $\mathrm{R}_{\mathrm{J}}$ by instability, and then both diffuse outward together. It has been shown by many authors that instability plays a major role in the outward diffusion of $\mathrm{O}^{+}$(Southwood \& Kivelson, 1987; Ma et al., 2016). Figure 5 shows that the region where Io plasma diffuses outward due to such instability spreads within $20 \mathrm{R}_{\mathrm{J}}$. Similar structure (interchange fingers) is calculated by Winglee et al. (2013), but the shape of fingers seems to be slightly different. In particular, Figure 5 is characterized by fingers extending in the longitude direction. This structure indicates that exchange occurs with the counterclockwise rotation as seen from the north. The direction of rotation can be reversed, but mixing of rotation direction seems not to occur. In addition, speed of movement in the longitude direction by planetary rotation seems faster than speed of interchange between inside and outside due to instability.

Next, looking at pink lines, magnetic field lines at the low-latitude boundary of the main oval extend to around $20 \mathrm{R}_{\mathrm{J}}$. In addition, there is almost no longitudinal bending in pink field lines. Comparing gold contour lines and pink magnetic field lines, the torus region of $160 \mathrm{amu} / \mathrm{cc}$ that extends as far as $12 \mathrm{R}_{\mathrm{J}}$ is still more inside than the mapping region of the main oval. Here, the dipole magnetic field has not been significantly deformed yet. Furthermore, the area of $20 \mathrm{amu} / \mathrm{cc}$ finally extends to the vicinity of mapping point from the low-latitude edge of the main oval. That is, the main part of the Io torus (the region denser than $1 / 10$ of the maximum density) is more inside the mapping region of the main oval. The outer edge of the Io disk (the area where the density is less than $1 / 20$ of the peak) finally extends to the mapping area of the low-latitude boundary of the main oval. It can be concluded that the main body of the Io disk is more inside than the region where the electrodynamic process works for the generation of the main aurora. This structure is the same as that derived from the simulation of Chané et al. (2013).

In the ionosphere, the equatorial edge of the main oval is $15.7^{\circ}$ colatitude (Figure 3 ). In Figure 5 , this position is mapped around $20 \mathrm{R}_{\mathrm{J}}$. For the dipole, mapping from $13^{\circ}$ colatitude leads to the observed starting position of corotation delay $\left(20 \mathrm{R}_{\mathrm{J}}\right)$. If the dipole is not modified, the low-latitude edge of the main aurora, $15.7^{\circ}$ colatitude, should be mapped within the dipole region which is almost completely corotating. Therefore, in Figure 5, magnetic field lines are stretched more than the dipoles, and the Io disk becomes on the lower-latitude side of the oval. From these mappings, it is estimated in this paper that there is a region within $20 R_{J}$ 


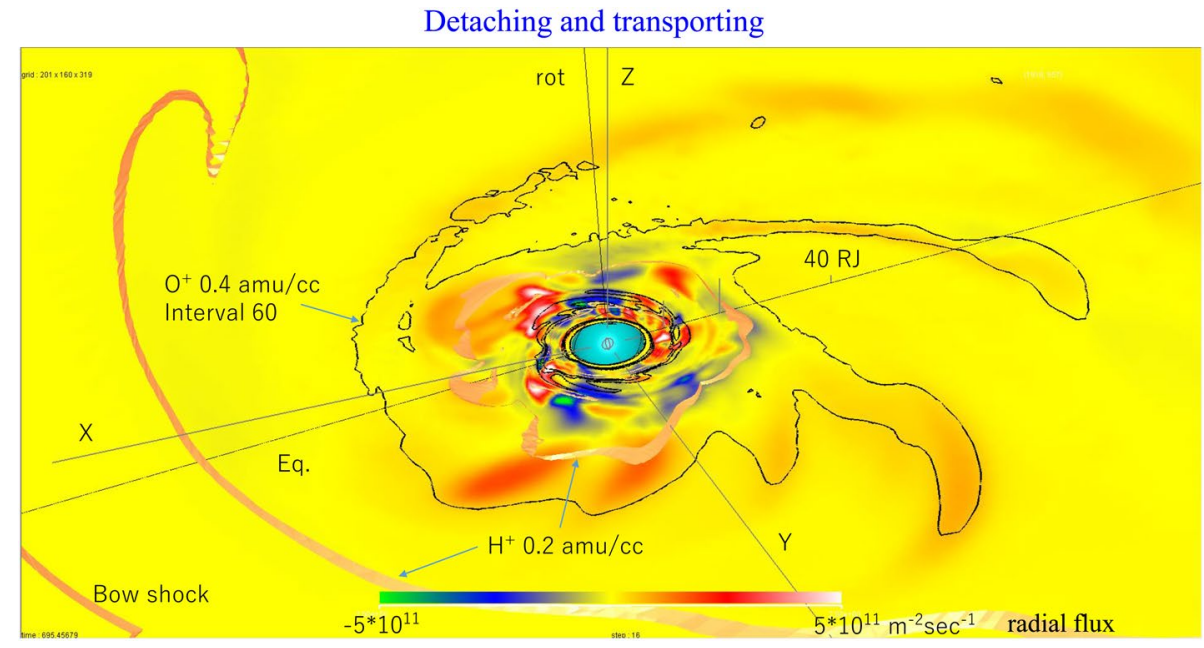

Figure 6. Distributions of $\mathrm{O}^{+}$(black contours) and $\mathrm{H}^{+}$(gold contours) mass densities and radial mass flux (color shadings) in the equatorial plane of the rotational axis coordinate. Transporting process due to instability and centrifugal force, and detaching process of plasmoid are visible in this figure.

where instability acts on the Io plasma disk to promote outward diffusion and the FAC is supplied to the region outside of it where the FAC reduces the corotation delay.

\subsection{Ejection of Plasmoid and Back Reaction}

Figure 2 shows that the high-latitude edge of the main oval is adjacent to the open magnetic field line region of the lobe, and Figure 5 shows that the low-latitude edge of the main oval is adjacent to the high-latitude edge of the Io disk. Taken together, we can conclude that magnetic field lines connected to the main oval are closed and projected onto the entire plasma sheet outside the Io disk. The FAC is almost absent inside the low-latitude edge of the main oval. Such a distribution of the FAC indicates outward ejection of Io plasma trough the two-step radial diffusion as shown below. The magnetic tension in the plasma disk is so strong that the centrifugal force cannot overcome the magnetic tension yet. Therefore, diffusion due to the exchange instability prevails there. That is, inside the Io disk, plasma diffuses outward due to the instability (first stage). Beyond $20 \mathrm{R}_{\mathrm{J}}$, on the contrary, the plasma can move outward by coupling with the FAC system. Whereas the rotational motion is maintained by acquiring angular momentum through the FAC, the centrifugal force becomes stronger as the increase of radial distance. On the other hand, the farther the distance is, the weaker the magnetic tension becomes. As a result, plasma cannot be retained to appropriate distance. Magnetic field line with weak magnetic tension causes elongation and deflection, associated with formation of the FAC system (second stage).

While the first stage of transport can be seen in Figure 5, the second stage can be seen in Figure 6. In Figure 6, black contour lines show the $\mathrm{O}^{+}$distribution on the equatorial plane of the rotation axis coordinate, gold contours show the similar $\mathrm{H}^{+}$distribution, and color shadings show the radial flux distribution. Movie 2 of supporting information is a movie of Figure 6. It can be clearly seen in Movie 2 that the contour structure rotates with the planet, ion distributions deform along with the rotation, the rotational feature spreads over the entire plasma sheet, and $\mathrm{O}^{+}$arcs extends outward. The winding structure of the plasma distribution associated with rotation corresponds to white magnetic field lines in Figure 1. Such rotationally driven convection occupies a wide area, and the Dungey cycle does not appear to be major in the Jupiter plasma sheet.

When magnetic field lines become bending arcs, they cannot stretch infinitely. So at certain moment, magnetic field and plasma are detached from the rotating structure. Also, due to the influence of the solar wind, the dayside magnetosphere is compressed and restricted. Due to a narrow region on the dayside, it is difficult for magnetic field line extending long on the nightside to pass through from the morning region to the evening region associated with rotation. In such a structure, it is necessary to separate a tip part of the arc 


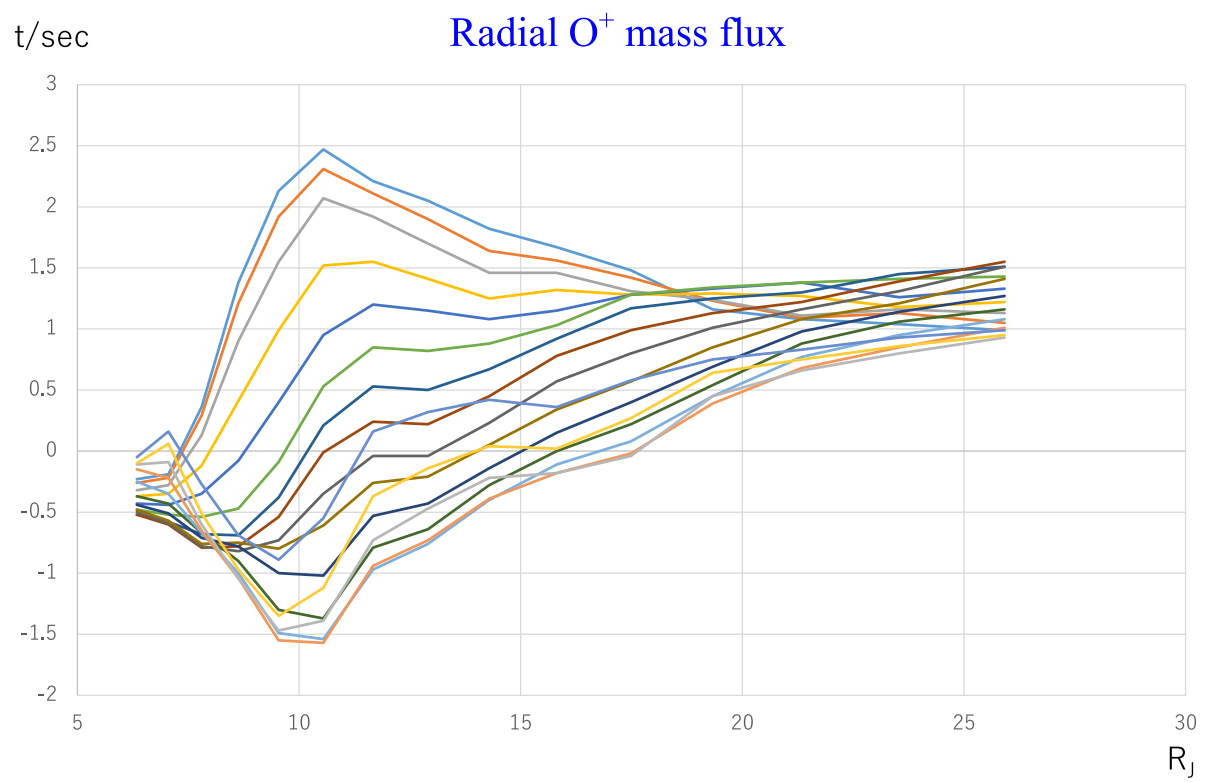

Figure 7. Radial distributions of radial $\mathrm{O}^{+}$mass flux at various timings. One curve represents the radial distribution at a particular time of the flux passing through spheres of respective radii.

in order for magnetized plasma extending on the nightside to slide into the dayside. For these two reasons, plasma sheet rotation needs to accompany the reconnection and plasmoid ejection.

The feature of plasmoid release can also be observed in Movie 2 of the supporting information. Contours in Figure 6 and Movie 2 do not necessarily illustrate the center of the plasma sheet in the distant region, but they still present features like plasmoids. The rotational motion from Jupiter is transmitted to the entire plasma sheet, and ejection of plasmoids occurs accordingly. In terms of composition, plasmoids are mixed plasma. In this way, the structure of magnetospheric convection at Jupiter is different from that of the Earth. The morphology of plasmoid release in Movie 2 is similar to that of Fukazawa et al. (2006). The number of plasmoid release is once every two days in Fukazawa et al. (2006), but in this calculation it is once in half a day. Even after the contraction due to the release of plasmoid, the arc hits the magnetopause on the day side, causing fluctuations in the position of the magnetopause.

In Figure 6 and Movie 2, the plasmoid develops associated with upwelling flow (red to white color). The arc begins to grow in the dayside region with strong upwelling. Entering the nightside, the arc elongates and then ejects out together with an upwelling flow. In the aftermath of the plasmoid ejection on the nightside, not only the $\mathrm{O}^{+}$density but also the velocity dynamically fluctuates in the plasma sheet. It is noteworthy that downward flow (blue to green color) is generated on the opposite side of the ejecting plasmoid. While striped patterns of upward and downward flows due to the exchange instability exist at the radial distance of $10 \mathrm{R}_{\mathrm{J}}$, striped patterns are greatly distorted when downward flow occurs due to the release of the plasmoid. This feature indicates that the first-stage transport is deformed and affected by the second-stage transport. Therefore, transport in the first stage is not stationary but fluctuates due to the reaction of plasmoid release.

Figure 7 gives an overview of radial transport. In Figure 7, the total amount of outward $\mathrm{O}^{+}$flux passing through a sphere is calculated for different radii, and then Figure 7 draws this flux value as the vertical axis with the radius of spheres as the horizontal axis. One curve is the radial distribution at a certain time, and Figure 7 draws curves over one rotation of Jupiter together. The radial distribution of the flux is dynamic and fluctuates with time. The most volatile point is $10 \mathrm{R}_{\mathrm{J}}$ which is the central position of transportation caused by the instability effect. Beyond $20 \mathrm{R}_{\mathrm{J}}$, where centrifugal force acts the main role, transport value approaches a certain value. At some timing, strong downward flow may predominate throughout the domain. This structure indicates that $\mathrm{O}^{+}$is lost not only by ejecting into the plasma sheet but also by falling into the ionosphere. The average amount of $\mathrm{O}^{+}$lost up and down is approximately the same as the amount of plasma produced by Io. It can be interpreted that the entire radial transport throughout the first and second 


\section{$\mathrm{O}^{+} \mathrm{H}^{+}$radial distributions}

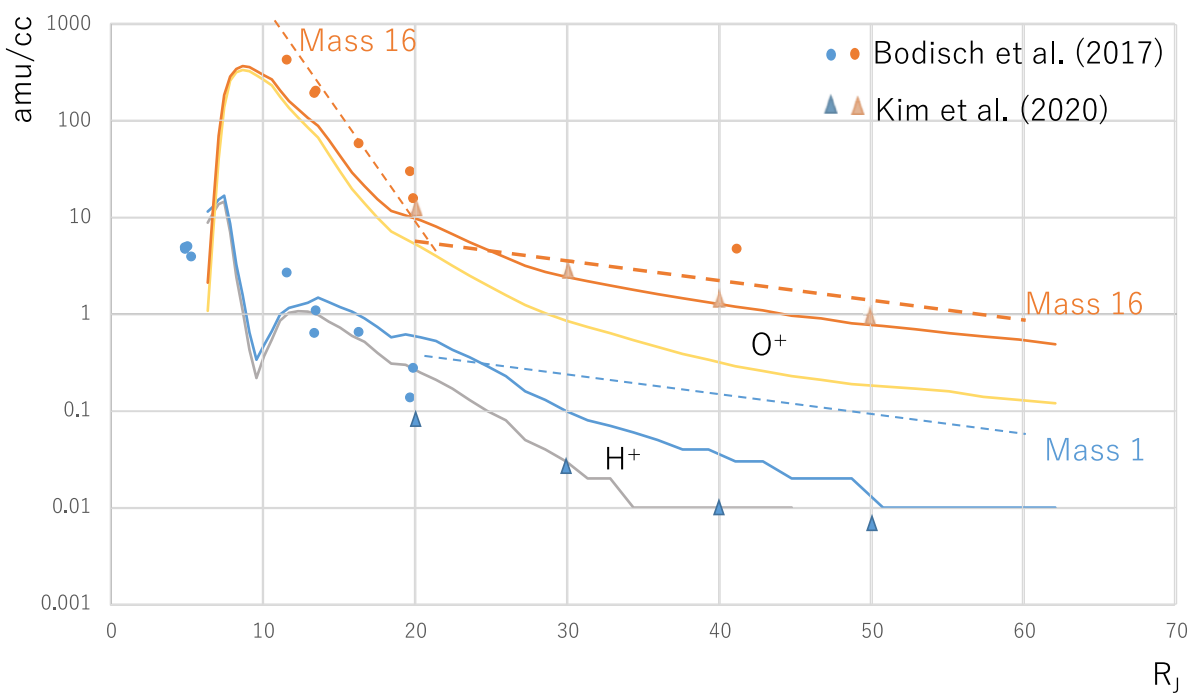

Figure 8. Calculated (solid lines) and observed (dashed lines, triangles, and dots) radial distributions of $\mathrm{O}^{+}$and $\mathrm{H}^{+}$. Here, amu/cc value marked by $\mathrm{O}^{+}$represents the sum of heavy ions. Upper lines (orange and blue) show local time means of latitudinal maximum, and lower lines (yellow and gray) show local time means of latitudinal mean over $\pm 8^{\circ}$ of the equatorial plane in the rotation axis coordinate. Dots show Voyajer observations (Bodisch et al., 2017), triangles show Juno observations (Kim et al., 2020), and dashed lines show mass distributions estimated from observation of electron density.

stages fluctuates due to the counter effect of plasmoid release. The counter reaction extends even to the first stage transport, and Figure 7 is the final result by summarizing them.

If the main oval is mapped to the entire plasma sheet, the downward FAC that is the return current of the upward FAC cannot be inside the plasma sheet. Therefore, it would be inappropriate to draw the return downward FAC from the outer edge of the Io disk, as shown in the schematic diagram of Cowley, Bunce and Nichols (2003). It would be more appropriate to consider the FAC closure via the distant magnetopause far from the plasma sheet, as in the schematic diagram of Cowley et al. (2005) and Chané et al. (2013). In this case, the FAC closure in the magnetosphere will occur over a wide range in the magnetosphere. However, as expected from the magnetic field structure in Figure 1, the downward FAC in the polar cap will not come from the magnetopause in the immediate vicinity, but will rather connect to far distant magnetotail. Such a current system is reproduced by the simulation in Moriguchi et al. (2008).

\subsection{Comparison With Observations}

Figure 8 shows the mass density distributions of $\mathrm{O}^{+}$and $\mathrm{H}^{+}$with respect to the distance from Jupiter, throughout the Io plasma disk and the mid-tail plasma sheet. Solid orange and yellow lines show calculated mass density distribution of $\mathrm{O}^{+}$, and blue and gray lines show the calculated mass density distribution of $\mathrm{H}^{+}$. Two values are shown for each ion at a certain $\mathrm{R}$ (radius). The first (lower) is the average value over latitudes $\pm 8^{\circ}$ of the equatorial plane of the rotation axis coordinate and over local time from 0 LT to 24 LT. The second (upper) is the value obtained by first taking the maximum in the latitude region $\pm 8^{\circ}$ from equatorial plane and then averaging over local time. The mass density distribution is always accompanied by time fluctuations. Figure 8 captures a moment, and small fluctuations are always superimposed on it at the moment to moment. In mass density distributions of $\mathrm{O}^{+}$and $\mathrm{H}^{+}, \mathrm{O}^{+}$always has a larger amount than $\mathrm{H}^{+}$both in the Io plasma disk and in the plasma sheet. Within $20 \mathrm{R}_{\mathrm{J}}$, the difference between the first and second values is still small. This result indicates that the Io disc is still thick. The difference gradually widens beyond $20 \mathrm{R}_{\mathrm{J}}$, and there is a five-fold difference between the two in the tail. This difference indicates that the thickness of the plasma sheet in this region is $1 / 5$ of $16^{\circ}$ which is about $3^{\circ} . \mathrm{H}^{+}$is not the main component in either the Io disk or the plasma sheets. The distribution of $\mathrm{H}^{+}$in Figure 8 has a minimum at $10 \mathrm{R}_{\mathrm{J}}$ around the $\mathrm{O}^{+}$peak, since $\mathrm{H}^{+}$distributes there along the magnetic field lines at high latitudes (Figure 4). 
Next, calculated distributions of $\mathrm{H}^{+}$and $\mathrm{O}^{+}$mass densities are compared with observations. Orange and blue dots in Figure 8 are mass density observations of $\mathrm{O}^{+}$and $\mathrm{H}^{+}$by Voyager (Bodish et al., 2017). Voyager observed heavy ions such as $\mathrm{O}^{++}, \mathrm{O}^{+}, \mathrm{S}^{+++}, \mathrm{S}^{++}, \mathrm{S}^{+}$, and $\mathrm{Na}^{+}$. The value displayed in Figure 8 as $\mathrm{O}^{+}$is the sum of them. Observed amu per charge varies depending on the altitude, and is about 26 at low altitudes and about 15 at high altitudes. Orange and blue triangles in Figure 8 are mass density observations of $\mathrm{O}^{+}$, $\mathrm{S}^{++}$, and $\mathrm{H}^{+}$(blue) by Juno (Kim et al., 2020). The value displayed by orange triangles in Figure 8 as $\mathrm{O}^{+}$is the sum of $\mathrm{O}^{+}, \mathrm{S}^{+}, \mathrm{S}^{++}$and $\mathrm{S}^{+++}$. Dashed lines are simplified distributions inferred from observations of electron density (Bagenal \& Delamere, 2011; Frank et al., 2002). Observed value of mass density in the tail inferred from electron density is orange dashed lines if all ions are $\mathrm{O}^{+}$, and a blue dashed line if all ions are $\mathrm{H}^{+}$. This simplified value is close to the value used in Chane et al. (2013) for the evaluation of simulation results. It can be regarded as the average of observed values shown in several papers.

Dots and dashed lines obtained by observations continue almost smoothly without a contradiction. In the observation in the tail, it is not clear at what ratio the plasma sheet interior and the place slightly deviated from it were scanned, so it is appropriate to do comparison by using the middle of first and second values obtained by the calculation. In the observation (orange dots and dashed line), the maximum value of mass density reaches to $1,600 \mathrm{amu} / \mathrm{cc}$ or more within $10 \mathrm{R}_{\mathrm{J}}$. This corresponds to $\mathrm{O}^{+}$number density of $100 / \mathrm{cc}$ or more. Since the position of the Io plasma generation (source) region is not so correct in the present calculation that the maximum value within $10 \mathrm{R}_{\mathrm{J}}$ may not match observations sufficiently (the calculated $\mathrm{O}^{+}$is less than the observation). At $10 \mathrm{R}_{\mathrm{J}}$, the observed mass density is $320 \mathrm{amu} / \mathrm{cc}$, and it corresponds to number density 20/cc if ions are $\mathrm{O}^{+}$. This value is considerably reproduced in calculation. Around the Io plasma disk from $10 \mathrm{R}_{\mathrm{J}}$ to outside, the decreasing feature of $\mathrm{O}^{+}$in the calculated result reproduces the observed decreasing rate fairly well. From this feature, it can be estimated that the diffusion process has been reproduced to some extent. In the simulation results of Sarkango et al. (2019), the decreasing slope is more gradual than observations and appears to be more diffusive. Beyond $35 \mathrm{R}_{\mathrm{J}}$ in the tail, calculated $\mathrm{O}^{+}$mass density is slightly less than Voyager's observations. Estimating from the electron density under an assumption that all ions are $\mathrm{O}^{+}$(orange line), the calculated value is slightly less than the observed value. This is also the case with results of Chané et al., (2013).

In the distribution of $\mathrm{H}^{+}$, the mass density and the number density are the same. In the disk region in Figure 8, the calculated result for $\mathrm{H}^{+}$reproduces the Voyager observation shown by blue dots. The observation by Cohen et al. (2001) show that the composition near 10-15 $R_{\mathrm{J}}$ becomes more iogenic. The calculated result is consistent with such observation also. Beyond $40 \mathrm{R}_{\mathrm{J}}$, observed $\mathrm{H}^{+}$mass density is below $0.1 / \mathrm{cc}$ (Bodisch et al., 2017), which is consistent with calculated values. Farther away $\left(\sim 600 R_{\mathrm{J}}\right)$ in the plasma sheet, observations show that $\mathrm{H}^{+}$becomes the major ion component (McComas et al., 2017). However, this observation will not be reproduced even if the results in Figure 8 are extended to the far tail region. In this region, observed ions $\left(\mathrm{H}^{+}\right)$are presumed to originate not only from Jupiter but also from the solar wind.

\section{Conclusions}

From the global simulation, we reproduced the solar wind-magnetosphere-ionosphere interaction at Jupiter. The model includes planetary rotation, Io plasma emission, ionospheric plasma, and auroral conductivity in the ionosphere. Simulation results reproduce magnetic configuration of the rotating magnetosphere, the Io plasma disk, disk instability, formations of the plasma sheet, plasmoid ejection, and generations of the FAC and aurora. In number density, $\mathrm{O}^{+}$is the main constituent of the Io plasma disk and the plasma sheet. While plasma density of the Io disk and the plasma sheet depend on the amount of Io plasma emission, magnetospheric $\mathrm{H}^{+}$density does not depend much on the solar wind.

Io plasma near 10-20 $R_{J}$ diffuses outward due to the instability effect and beyond $20 R_{J}$ moves outward by centrifugal force with stretch of magnetic field. The iogenic $\mathrm{O}^{+}$and the ionospheric $\mathrm{H}^{+}$are mixed at the outer edge of the rotating disk around $15-20 R_{\mathrm{J}}$ (density is about $1 / 100$ of the maximum). Then, mixed plasma diffuses farther outward transmitting internal driven convection. Due to the limit of magnetic field stretching, the plasmoid ejection occurs in the nightside plasma sheet and mixed plasma spreads over the entire equatorial plane in the tail. Counter downward flow associated with plasmoid ejection even affects the instability in the Io disk and causes modulation for radial plasma transport in the entire system. 
In the ionosphere, the main oval occurs over colatitudes from $15.7^{\circ}$ to $13.5^{\circ}$. The main part of the rotating Io plasma disk is more inside than the mapping area of the main oval. The main oval is mapped to the entire plasma sheet. The global current system that exchange rotation motion between the ionosphere and the magnetosphere constructs from the upward FAC in the main oval, ionospheric current, and downward FAC in the polar cap. However, upward and downward FACs are not closed in the near-Jupiter magnetosphere. The downward FAC shows rapid fluctuation similar to the swirl. Due to the presence of twisted magnetic field lines throughout the entire polar cap, externally driven Dungey convection can occur only in a narrow gap at the boundary between the main oval and the polar cap.

\section{Data Availability Statement}

Numerical data https://doi.org/10.17592/002.2020100389, coordinate data, and variable data, information for graphic program and history data necessary to reproduce drawings are available at https://scidbase.nipr.ac.jp/modules/metadata/index.php?content_id=389/ and at the rink from it http://polaris.nipr. ac.jp/ reppu/reppu2/2020c_jov_v02/.

Acknowledgments

In the present study, we used the highspeed computing system at Polar Data Center of National Institute of Polar Research through General Collaboration Project 2-3, the KDK computer system at the Research Institute for Sustainable Humanosphere (RISH), Kyoto University through General Collaboration Project R2-KDK-03, and Nagoya University super computer system through Nagoya University High Performance Computing Research Project for Joint Computational Science in Japan. This study was supported by KAKENHI (JSPS KAKENHI Grant Number JP20H01960, YE) and KAKENHI (JSPS KAKENHI Grant Number JP20K03894, MW).

\section{References}

Achilleos, N., Dougherty, M. K., Young, D. T., \& Crary, F. (2004). Magnetic signatures of Jupiter's bow shock during the Cassini flyby. Journal of Geophysical Research, 109, A09S04. https://doi.org/10.1029/2003JA010258

Bagenal, F., \& Delamere, P. A. (2011). Flow of mass and energy in the magnetospheres of Jupiter and Saturn. Journal of Geophysical Research, 116, A05209. https://doi.org/10.1029/2010JA016294

Bodisch, K. M., Dougherty, L. P., \& Bagenal, F. (2017). Survey of voyager plasma science ions at Jupiter: 3. Protons and minor ions. Journal of Geophysical Research: Space Physics, 122, 8277-8294. https://doi.org/10.1002/2017JA024148

Bunce, E. J., Cowley, S. W. H., \& Yeoman, T. K. (2004). Jovian cusp processes: Implications for the polar aurora. Journal of Geophysical Research, 109, A09S13. https://doi.org/10.1029/2003JA010280

Chané, E., Saur, J., Keppens, R., \& Poedts, S. (2017). How is the Jovian main auroral emission affected by the solar wind? Journal of Geophysical Research: Space Physics, 122, 1960-1978. https://doi.org/10.1002/2016JA023318

Chané, E., Saur, J., \& Poedts, S. (2013). Modeling Jupiter's magnetosphere: Influence of the internal sources. Journal of Geophysical Research: Space Physics, 118, 2157-2172. https://doi.org/10.1002/jgra.50258

Cohen, C. M. S., Stone, E. C., \& Selesnick, R. S. (2001). Energetic ion observations in the middle Jovian magnetosphere. Journal of Geophysical Research, 29, 871-881. https://doi.org/10.1029/2001JA000008

Cowley, S. W. H., Alexeev, I. I., Belenkaya, E. S., Bunce, E. J., Cottis, C. E., Kalegaev, V. V., et al. (2005). A simple axisymmetric model of magnetosphere-ionosphere coupling currents in Jupiter's polar ionosphere. Journal of Geophysical Research, 110, A11209. https://doi. org/10.1029/2005JA011237

Cowley, S. W. H., Bunce, E. J., \& Nichols, J. D. (2003). Origins of Jupiter's main oval auroral emissions. Journal of Geophysical Research, 108(A4), 8002. https://doi.org/10.1029/2002JA009329

Cowley, S. W. H., Bunce, E. J., Stallard, T. S., \& Miller, S. (2003). Jupiter's polar ionospheric flows: Theoretical interpretation. Geophysical Research Letters, 30(5), 1220. https://doi.org/10.1029/2002GL016030

Delamere, P. A., \& Bagenal, F. (2003). Modeling variability of plasma conditions in the Io torus. Journal of Geophysical Research, 108(A7), 1276. https://doi.org/10.1029/2002JA009706

Delamere, P. A., Otto, A., Ma, X., Bagenal, F., \& Wilson, R. J. (2015). Magnetic flux circulation in the rotationally driven giant magnetospheres. Journal of Geophysical Research: Space Physics, 120, 4229-4245. https://doi.org/10.1002/2015JA021036

Dunn, W. R., Gray, R., Wibisono, A. D., Lamy, L., Louis, C., Badman, S. V., et al. (2020). Comparisons between Jupiter's X-ray, UV and radio emissions and in-situ solar wind measurements during 2007. Journal of Geophysical Research: Space Physics, 125, e2019JA027222. https://doi.org/10.1029/2019JA027222

Frank, L. A., Paterson, W. R., \& Khurana, K. K. (2002). Observations of thermal plasmas in Jupiter's magnetotail. Journal of Geophysical Research, 107, 1003. https://doi.org/10.1029/2001JA000077

Fukazawa, K., Ogino, T., \& Walker, R. J. (2006). Configuration and dynamics of the Jovian magnetosphere. Journal of Geophysical Research, 111, A10207. https://doi.org/10.1029/2006JA011874

Grodent, D., Clarke, J. T., Kim, J., Waite, J. H., Jr, \& Cowley, S. W. H. (2003). Jupiter's main auroral oval observed with HST-STIS. Journal of Geophysical Research, 108(A11), 1389. https://doi.org/10.1029/2003JA009921

Grodent, D., Clarke, J. T., Waite, J. H., Jr, Cowley, S. W. H., Gérard, J.-C., \& Kim, J. (2003). Jupiter's polar auroral emissions. Journal of Geophysical Research, 108(A10), 1366. https://doi.org/10.1029/2003JA010017

Hanlon, P. G., Dougherty, M. K., Forsyth, R. J., Owens, M. J., Hansen, K. C., To'th, G., et al. (2004). On the evolution of the solar wind between 1 and $5 \mathrm{AU}$ at the time of the Cassini Jupiter flyby: Multispacecraft observations of interplanetary coronal mass ejections including the formation of a merged interaction region. Journal of Geophysical Research, 109, A09S03. https://doi.org/10.1029/2003JA010112

Hess, S. L. G., Bonfond, B., Zarka, P., \& Grodent, D. (2011). Model of the Jovian magnetic field topology constrained by the Io auroral emissions. Journal of Geophysical Research, 116, A05217. https://doi.org/10.1029/2010JA016262

Hill, T. W. (2001). The Jovian auroral oval. Journal of Geophysical Research, 106, 8101-8107. https://doi.org/10.1029/2000JA000302

Khurana, K. K., Kivelson, M. G., Vasyliunas, V. M., Krupp, N., Woch, J., Lagg, A., et al. (2004). The configuration of Jupiter's magnetosphere. In F. Bagenal, T. E. Dowling, \& W. B. McKinnon (Eds.), Jupiter. The planet, satellites and magnetosphere (pp. 593-616). Cambridge, U.K: Cambridge University Press.

Kidder, A., Winglee, R. M., \& Harnett, E. M. (2009). Regulation of the centrifugal interchange cycle in Saturn's inner magnetosphere. Journal of Geophysical Research, 114, A02205. https://doi.org/10.1029/2008JA013100 
Kim, T. K., Ebert, R. W., Valek, P. W., Allegrini, F., McComas, D. J., Bagenal, F., et al. (2020). Survey of ion properties in Jupiter's plasma sheet: Juno JADE-I observations. Journal of Geophysical Research: Space Physics, 125, e2019JA027696. https://doi.org/10.1029/2019JA027696

Kimura, T., Hiraki, Y., Tao, C., Tsuchiya, F., Delamere, P. A., Yoshioka, K., et al. (2018). Response of Jupiter's aurora to plasma mass loading rate monitored by the Hisaki satellite during volcanic eruptions at Io. Journal of Geophysical Research: Space Physics, 123, $1885-1899$. https://doi.org/10.1002/2017JA025029

Kivelson, M. G., \& Southwood, D. J. (2005). Dynamical consequences of two modes of centrifugal instability in Jupiter's outer magnetosphere. Journal of Geophysical Research, 110, A12209. https://doi.org/10.1029/2005JA011176

Ma, X., Delamere, P. A., \& Otto, A. (2016). Plasma transport driven by the Rayleigh-Taylor instability. Journal of Geophysical Research: Space Physics, 121, 5260-5271. https://doi.org/10.1002/2015JA022122

McComas, D. J., Allegrini, F., Bagenal, F., Ebert, R. W., Elliott, H. A., Nicolaou, G., et al. (2017). Jovian deep magnetotail composition and structure. Journal of Geophysical Research: Space Physics, 122, 1763-1777. https://doi.org/10.1002/2016JA023039

Miyoshi, T., \& Kusano, K. (2001). A Global MHD simulation of the Jovian magnetosphere interacting with/without the interplanetary magnetic field. Journal of Geophysical Research, 106, 10723-10742. https://doi.org/10.1029/2000ja900153

Moriguchi, T., Nakamizo, A., Tanaka, T., Obara, T., \& Shimazu, H. (2008). Current systems in the Jovian magnetosphere. Journal of Geophysical Research, 113, A05204. https://doi.org/10.1029/2007JA012751

Nichols, J. D., \& Cowley, S. W. H. (2003). Magnetosphere-ionosphere coupling currents in Jupiter's middle magnetosphere: Dependence on the effective ionospheric Pedersen conductivity and iogenic plasma mass outflow rate. Annales Geophysicae, 21, 1419-1441. https:// doi.org/10.5194/angeo-21-1419-2003

Nichols, J. D., \& Cowley, S. W. H. (2004). Magnetosphere-ionosphere coupling currents in Jupiter's middle magnetosphere: Effect of precipitation-induced enhancement of the ionospheric Pedersen conductivity. Annales Geophysicae, 22, 1799-1827. https://doi.org/10.5194/ angeo-22-1799-2004

Paranicas, C., Mauk, B. H., Haggerty, D. K., Clark, G., Kollmann, P., Rymer, A. M., et al. (2018). Intervals of intense energetic electron beams over Jupiter's poles. Journal of Geophysical Research: Space Physics, 123, 1989-1999. https://doi.org/10.1002/2017JA025106

Pu, Z.-Y., \& Kivelson, M. G. (1983). Kelvin-helmholtz instability at the magnetopause: Energy flux into the magnetosphere. Journal of Geophysical Research, 88, 853-861. https://doi.org/10.1029/JA088iA02p00853

Radioti, A., Krupp, N., Woch, J., Lagg, A., Glassmeier, K.-H., \& Waldrop, L. S. (2005). Ion abundance ratios in the Jovian magnetosphere. Journal of Geophysical Research, 110, A07225. https://doi.org/10.1029/2004JA010775

Sarkango, Y., Jia, X., \& Toth, G. (2019). Global MHD simulations of the response of Jupiter's magnetosphere and ionosphere to changes in the solar wind and IMF. Journal of Geophysical Research: Space Physics, 124, 5317-5341. https://doi.org/10.1029/2019JA026787

Smyth, W. H., Peterson, C. A., \& Marconi, M. L. (2011). A consistent understanding of the ribbon structure for the Io plasma torus at the Voyager 1, 1991 ground-based, and Galileo J0 epochs. Journal of Geophysical Research, 116, A07205. https://doi.org/10.1029/2010JA016094

Southwood, D. J., \& Kivelson, M. G. (1987). Magnetospheric interchange instability. Journal of Geophysical Research, 92, 109-116. https:// doi.org/10.1029/JA092iA01p00109

Tanaka, T. (1994). Finite volume TVD scheme on an unstructured grid system for three-dimensional MHD simulation of inhomogeneous systems including strong background potential fields. Journal of Computational Physics, 111(2), 381-389. https://doi.org/10.1006/ jcph.1994.1071

Tanaka, T., Ebihara, Y., Watanabe, M., Den, M., Fujita, S., Kikuchi, T., et al. (2017). Global simulation study for the time sequence of events leading to the substorm onset. Journal of Geophysical Research: Space Physics, 122, 6210-6239. https://doi.org/10.1002/2017JA024102

Tanaka, T., Ebihara, Y., Watanabe, M., Den, M., Fujita, S., Kikuchi, T., et al. (2020). Reproduction of ground magnetic variations during the SC and the substorm from the global simulation and biot-savart's law. Journal of Geophysical Research: Space Physics, 125, e2019JA027172. https://doi.org/10.1029/2019JA027172

Tanaka, T., \& Murawski, K. (1997). Three-dimensional MHD simulation of the solar wind interaction with the ionosphere of Venus: Results of two-component reacting plasma simulation. Journal of Geophysical Research, 102, 19805-19821. https://doi.org/10.1029/97ja01474

Tanaka, T., Watanabe, M., Den, M., Fujita, S., Ebihara, Y., Kikuchi, T., et al. (2016). Generation of field-aligned current (FAC) and convection through the formation of pressure regimes: Correction for the concept of Dungey's convection. Journal of Geophysical Research: Space Physics, 121, 8695-8711. https://doi.org/10.1002/2016JA022822

Vasyliunas, V. M. (1983). Plasma distribution and flowPhysics of the Jovian magnetosphere, chap. Plasma distribution and flow (pp. 395-453). Cambridge, New York: Cambridge Planetary Science Series. https://doi.org/10.1017/cbo9780511564574.013

Vogt, M. F., Bunce, E. J., Nichols, J. D., Clarke, J. T., \& Kurth, W. S. (2017). Long-term variability of Jupiter's magnetodisk and implications for the aurora. Journal of Geophysical Research: Space Physics, 122, 12090-12110. https://doi.org/10.1002/2017JA024066

Vogt, M. F., Kivelson, M. G., Khurana, K. K., Walker, R. J., Bonfond, B., Grodent, D., \& Radioti, A. (2011). Improved mapping of Jupiter's auroral features to magnetospheric sources. Journal of Geophysical Research, 116, A03220. https://doi.org/10.1029/2010JA016148

Walker, R. J., \& Ogino, T. (2003). A simulation study of currents in the Jovian magnetosphere. Planetary and Space Science, 51, $295-307$. https://doi.org/10.1016/s0032-0633(03)00018-7

Winglee, R. M., Kidder, A., Harnett, E., Ifland, N., Paty, C., \& Snowden, D. (2013). Generation of periodic signatures at Saturn through Titan's interaction with the centrifugal interchange instability. Journal of Geophysical Research: Space Physics, 118, 4253-4269. https:// doi.org/10.1002/jgra.50397

Wu, H., Hill, T. W., Wolf, R. A., \& Spiro, R. W. (2007). Numerical simulation of fine structure in the Io plasma torus produced by the centrifugal interchange instability. Journal of Geophysical Research, 112, A02206. https://doi.org/10.1029/2006JA012032

Zhang, B., Delamere, P. A., Yao, Z., Bonfond, B., Lin, D., Sorathia, K. A., et al. (2021). How Jupiter's unusual magnetospheric topology structures its aurora. Science Advances, 7(15), eabd1204. https://doi.org/10.1126/sciadv.abd1204 